\title{
Ref 2014 and Impact Reading the Runes for Anthropology in Action
}

\section{Bob Simpson}

On 18 December 2014, the results of the U.K.'s Research Excellence Framework (REF) evaluation exercise were released. This extensive and very costly exercise is intended to take the pulse of U.K. university-based research and now happens once every six years or so. It is also the principal tool used to determine the allocation of approximately $£ 1.6$ billion of quality-related $(\mathrm{QR})$ research funding which maintains the fabric of research activity in U.K. HE institutions. Given the fiscal consequences of REF performance it is not surprising that that universities expended considerable time and effort preparing their submissions in the run-up to the exercise and that the results were pored over by academics and their managers across the country. This was a very complex set of runes to read.

The 2014 exercise was made rather more complex by the fact that for the first time 20 per cent of the overall scoring of a unit of assessment (UoA), and hence a portion of $\mathrm{QR}$ funding to universities, was attributed to research 'impact'. Through a combination of 'impact case studies' (ICSs) based on specific research and an 'impact template' intended to capture the UoA's overall strategy, each REF panel would evaluate the consequences of research activity beyond the academy. A definition was provided of what actually constituted 'impact' ${ }^{1}$ and examples were given. For a community of intelligent, critical and often sceptical academics, just what to make of this apparent cuckoo in the nest provoked much debate and argument. However, what went on before REF 2014 is as nothing compared with what is happening in its wake. It would seem that a substantial sub-industry has sprung up as the impact of 'impact' begins to sink in. Put bluntly, under the present algorithm, without a strong set of impact case studies, a good overall score or Grade Point Average will be virtually impossible to achieve. Like it or loathe it, the impact agenda will be widely felt over the next six to eight years and with it will come careful reflection on anthropology that is 'applied', 'engaged', 'action-oriented', 'collaborative', 'participative' and a host of other terms which reference ways in which the subjects of our research are woven into the objects of knowledge that we eventually create.

These are issues that have long been core to Anthropology in Action's concerns. That the journal has taken it upon itself to devote a special issue to impact is thus an important initiative. Likewise, making 'impact' a standing item of content in the journal with editors Samantha Page and Natali Valdez dedicated to maintaining debate and dissemination on this topic is also to be welcomed. In this brief editorial I would like to sketch out why these initiatives are so important for the discipline. Before commencing with a reflection on the terms of the 'impact' debate as it now stands, however, it might be worth revisiting what actually happened in sub-panel UoA 24 Anthropology and Development when it came to impact assessment. ${ }^{2}$

The sub-panel assessed twenty-five 'impact templates' and eighty impact case studies (ICSs). All UoAs submitted an impact template and a minimum of two ICSs. Additional ICSs were required for every ten fulltime equivalent academic staff (FTEs) submitted above a baseline of 15FTEs. The numbers of ICSs submitted to UoA 24 (Anthropology and Development) ranged between two and seven per UoA. After much calibration and cross-referencing within and between panels, each template and case study was assessed by at least one 'impact assessor' and a panel member. Impact assessors were drawn from the worlds of policy and application. In this instance they came with anthropologically informed experience in DfID, the British Museum and the Scottish Government. All impact items were, in effect, double blind-marked with each score being carefully wrought before being put 
before the entire panel. Impact assessors played an important role in reviewing the evidence on which claims of impact rested and, moreover, applying the REF-given criteria of 'reach' and 'significance' to this evidence. In the event, 40.8 per cent of UoA 24 impact submissions were deemed to be $4^{*}$ (that is, 'outstanding' in terms of reach and significance) and 43.2 per cent were deemed to be $3^{*}$ (that is, 'very considerable' in terms of reach and significance). These figures compare with output scores of 19.1 per cent at $4^{*}$ and 39.3 per cent at $3^{*}$. Comparison with the other ten subjects in Main Panel C (in which the social sciences were mostly located) would suggest that as a discipline we did well on impact (ranked third for both proportion of $4^{*}$ and of $4^{*}+3^{*}$ ) but not so well on outputs (ranked $11^{\text {th }}$ for both $4^{*}$ and $\left.4^{*}+3^{*}\right)$. This pattern was one that appears to have been replicated across all disciplines - impact scored more highly than outputs (overall average of 3.24 and 2.90 out of 4 respectively). Where UoA 24 did not follow wider trends was in the extent of the divergence between impact and output scores and the effect this then had on rank orders. Writing in the THES about REF2014, Paul Jump, one of the journal's analysts, claimed that 'except at the margins, the established pecking order had not been overturned by impact's influence' (19-25 February, p. 36). What he was referring to here was the pattern in U.K. higher education for resources to be clustered in older and wealthier universities [known as the Russell Group] and, moreover, ones that are located in the 'golden triangle' of London, Oxford and Cambridge. Whether the rankings in Main Panel C place anthropology as a discipline at the 'margins' I am not sure, but it is clear that the inclusion of impact had significant effects on UoA 24 rankings. For example: the London School of Economics' anthropology department ranked first for outputs but joint twenty-third for impact, Brunel seventh for outputs but joint twenty-third for impacts, Queen's University Belfast fourteenth for outputs but first for impact; SOAS eighth for outputs but joint seventeenth for impact. When combined, output and impact scores produced a rank ordering that many found surprising (and here read 'a cause for rejoicing' or 'frustration and anger' depending on whether fortunes rose or fell because of impact scores); the established 'pecking order' had indeed been unsettled. For development submissions in UoA 24, impact scores tended to carry units upward in the rankings. This is not so surprising given that impact is what development studies projects usually aspire to achieve from their inception. But, it is also not surprising that impact would play a significant role in the final rankings across the unit of assessment given that it accounted for $20 \%$ of the overall grade - the equivalent of multiple individual outputs. In short, impact played an important role in shaping the 2015 rankings. In the next REF exercise, impact might disappear, stay at the same weighting or increase. The first two are unlikely. The inclusion of impact in REF 2014 has been hailed as a great success by the agencies leading U.K. higher education policy. ${ }^{3}$ Given that plans for REF 2014 were for impact to be weighted more highly than in fact it was, it is a reasonably safe bet that in the next REF it will count for more than the present 20 per cent.

What are anthropologists to make of 'impact'? If my own Department is anything to go by there was considerable scepticism. The root of the concerns lay in the fact that for anthropologists the impact agenda touches on the fundamentals of anthropological method and theory. One of the main reasons for this is that it puts the relationships we have with the people, societies, communities, institutions and organisations we research in a different light. As the final report from UoA 24 puts it, the impact exercise demonstrated a strong institutional commitment to taking 'the best findings of researchers back into the world from which they had first come'. ${ }^{4}$ Yet, this is, and always has been, a rather sensitive loop for anthropologists to negotiate and particularly so when tied in some way to the interests of governments and state agencies through funding policy. The impact agenda thus raised new concerns for many colleagues because it appeared to open a backdoor to things that are inimical to anthropological research and the values upon which it is based. The argument looks something like the following: as higher education becomes ever more firmly hitched to a neoliberal bandwagon, the value of everything, including research, becomes more closely indexed to the economy and to particular models of utility. The resulting instrumentalisation of research is believed to set in train a number of consequences: it introduces audit, quantification and measurement in ways that do not sit comfortably with open-ended and holistic approaches favoured by anthropologists; there are concerns about the prominence of 'behaviour change' in the objectives of impact; research necessarily ends up being constructed with certain ends in mind and long before we even embark on a study; it promotes utilitarianism and artificiality in the relationship with those among whom we research by designating them as 'beneficiaries', 'users' and 'stakeholders' in relation to the work we do; it ultimately threatens academic freedom and may shrink the space for critical scholarship. There is concern that within a one-size-fits-all world we will all become slaves to 'impact' and anthropological research 
will be reduced to a frenzy of para-ethnographic events geared to gathering evidence of impact.

Such concerns are not to be dismissed lightly. However, there is another reading and, whilst not wholly optimistic, it is one that is inclined to see opportunity rather than crisis in the rise and rise of impact. As suggested above, anthropologists, despite their scepticism, actually came out rather well within the impact exercise. My impression, from having read all of the anthropology ICSs, is that there is some extremely important and imaginative work going on (an example of a successful REF Impact Case Study is included in this issue) and that anthropological research is making a difference in areas that matter. The Anthropology and Development Panel reported particular strengths in: 'visual anthropology and material culture, political and legal anthropology, anthropology of development, environmental anthropology and biological anthropology' (Overview Report by Main Panel C. p. 99). I would suggest that one of the reasons why anthropologists were able to engage effectively with the impact agenda is that long-term relationships are deeply inscribed in the methods we use. In many instances, therefore, despite being dressed up in the conceptual language of billiard ball collisions and linear causalities, impact referenced something that was not vastly different from what was already being done by many anthropologists and particularly those already working with anthropology as a tool for activism, advocacy and social justice. In this sense, the impact agenda provided a channel for recognition and reward for a kind of anthropology that is widely espoused but which is often relegated to the murky realms of applied research. It also enabled others, who might have thought of their work as rather more solipsistic, to see that it had in fact been translated into benefits of one kind or another outside of the academy.

So, two cheers for impact?

Whilst embrace of the impact agenda by anthropologists is unlikely to be unconditional, I would suggest that there are things that are worthy of careful reflection. Among these, I would suggest, is an evaluation of what constitutes public benefit arising from what we do; efforts to stimulate and promote public awareness and debate about anthropology and the development of a more analytical appraisal of the role of anthropology in the public sphere. All of these are issues that have long been high on the agenda of $\mathrm{An}$ thropology in Action and the present issue marks the opening of a new chapter in the engagement between anthropology and its publics. The pages of Anthropology in Action are a particularly good place to identify, explore and debate issues thrown up by the impact agenda, such as: what constitutes benefit? How might benefits be evidenced? Can we retain the integrity of relationships in the face of demands for such evidence? How can benefits, once identified, be sustained in the longer term? Moreover, Anthropology in Action provides a strong platform from which to develop responses to the various calls and consultations emanating from HEFCE and which in the coming years will shape the impact agenda.

In a first step along this road, the editorial board of Anthropology in Action called for contributions to a special issue on the topic of impact and anthropology. The response was encouraging. Articles were received across a range of topics and the eventual selection focused on articles and case studies that discussed the topic of impact per se - how it is being defined and measured, how it has become a focus of audit and interest in research and the potential 'impact of impact' on anthropology as a discipline and its institutions. The special issue starts with an article by Abigail Baim-Lance and Cecilia Vindrola-Padros that, using their own research as a case study, argues for a wider concept of impact and one capable of incorporating reflection and innovation in how research is conducted. In similar vein, a case study by Andrew Russell and Sue Lewis reflects on their own attempts to document the impact of their research into smoking practices and tobacco use. They speculate on how the 'impact case study' constitutes a new documentary form that may in future shape how anthropologists approach their research. Their work highlights the need for a critical analysis to be undertaken by the anthropological community of the current methods of research evaluation and, more importantly, the need to formulate these into contributions to future consultations on REF methodologies. Michelle Stewart then looks at approaches to participatory working. Drawing on anthropological sensibilities and ethnographic practices, she describes how a wide range of 'frontline' workers in the field of foetal alcohol spectrum disorder (FASD) were brought together to participate in a workshop. Her article documents the ways in which her research made a difference in enabling different professionals to reflect on one another's practice and to devise ways of working together that would improve treatment and outcomes for those with FASD.

Returning to the theme of documenting and demonstrating impact, Neil Jarman and Dominic Bryan describe the anthropological research that formed the basis of their high-scoring impact case study. In a clear and straightforward exposition they illustrate how long-term, anthropological work on a 
specific topic (for example, the emotive topic of flags and parades in Northern Ireland) begins to have a much wider reach and significance than first envisaged. Taking a slightly different turn, a comment article by Grégory Dallemagne, Víctor del Arco, Ainhoa Montoya and Marta Pérez takes up the current debate about 'open access' in research. The authors take the debate beyond the narrow arguments about publishing practices to examine the implications of 'open access' for how research is conducted and communicated. The 'open access' question is of significance in this context because the next U.K. REF will require submitted publications to be open access and it is hard to imagine that this injunction would not also apply to impact-related publications. Finally, a meeting report is included from the fairly new but rapidly establishing EASA Network of Applied Anthropologists by the network's convenors, Dan Podjed and Meta Gorup. This is complemented by a review by Natali Valdez of a recent major U.K. publication - 'In Defence of Social Science' - which argues for the value of the social sciences in dealing with contemporary problems. I hope this issue will be widely read and will stimulate debate within and beyond anthropology about what impact means and how it might translate into the kinds of public benefit we would all wish to see.

Bob Simpson is a professor of anthropology at Durham University, U.K. In recent years his research interests have focused on the anthropology of bioethics, human subject research and new reproductive technologies. He served as a member on the REF Anthropology and Development panel (UoA 24) in 2014. E-mail: Robert.simpson@durham.ac.uk

For those interested in learning more about the U.K. impact exercise and the debates it has engendered see the following:

- All submitted impact case studies can be accessed through a searchable data base: http://impact.ref.ac.uk/ CaseStudies/

- London School of Economics has established a lively blog on the topic of impact at http://blogs.lse. ac.uk/impactofsocialsciences/?s=Bruce+Curtis
- Simon Bastow, Patrick Dunleavy and Jane Tinkler have produced a comprehensive overview: The Impact of the Social Sciences: How Academics and Their Research can make a Difference, published by Sage in 2015.

- There is a useful overview of audit and impact in different countries in a working paper by Wright et al.: http://edu.au.dk/en/research/research-areas/epoke/ publications/working-papers/

- See articles discussing the potential impact of the impact agenda on universities and research by Caroline Knowles and Roger Burrows: 'The Impact of Impact' and by Jon Mitchell: 'Anthropologists Behaving Badly? Impact and the Politics of Evaluation in an Era of Accountability', Etnográfica 18, no. 2: 237-54, 27597, June 2014.

\section{Notes}

1. On page 48 of the REF 2014: Assessment Framework and Guidance on Submissions impact is defined as: 'an effect on, change or benefit to the economy, society, culture, public policy or services, health, the environment or quality of life, beyond academia'.

2. The reduction in the overall number of UoAs assessed in the 2014 REF exercise meant that cognate disciplines [and in some cases not-so-cognate ones] were put into the same UoA. As a result, the exercise became highly strategic at an institutional level with some departments and many individual anthropologists finding themselves submitted to UoAs to which they were, on the face of it, quite marginal.

3. The whole exercise is currently being evaluated by RAND Europe but comments by David Sweeney, Director of Research and Knowledge Exchange at HEFCE reported in the THE suggest that the approach to impact 'worked effectively' (THE 19 February 2015 p. 38).

4. See page 99 of the Main Panel C Report, January 2015. http://www.ref.ac.uk/media/ref/content/expanel/ member/Main \%20Pane1\%20C\%20overview\%20 report.pdf 